\title{
Effects of viscosity and varying gravity on liquid sloshing in a carrier subjected to external excitations
}

\author{
Liming Dai · Xiaojie Wang
}

Received: 16 December 2013 / Revised: 20 February 2014 / Accepted: 22 February 2014 / Published online: 15 March 2014

(C) Springer-Verlag Berlin Heidelberg 2014

\begin{abstract}
The nonlinear dynamic behavior of liquid sloshing in a carrier is investigated in this research with consideration the effects of viscosity of the liquid and varying gravity on the carrier. Liquid sloshing in the tank of the carrier is analogized as a three-dimensional nonlinear conical pendulum model. The solutions of the coupled governing equations for the sloshing are developed and solved numerically. Both inviscid and viscous liquids are considered and compared for their effects on the sloshing. With the research results obtained, viscosity of the liquid plays a significant role in the nonlinear dynamic behavior of liquid sloshing. Also, the influence of gravitational acceleration on the 3D nonlinear sloshing of the liquid in the carrier is studied in details with a variety of system parameters.
\end{abstract}

Keywords Liquid sloshing - Conical pendulum model . Viscous liquid - Inviscid liquid - Gravitational acceleration . Nonlinear oscillation

\section{Introduction}

The phenomena of liquid sloshing in a tank extensively exist in industrial activities, such as those in liquid cargo transportation and spacecraft or rockets with liquid propellants. The sloshing liquid within tanks triggers forces and torques imposed on the tanks, which affect the motion of the liquid containers or vehicles and lead to unsafe operation and negative effects on their structural fatigue life. Analysis of liquid sloshing is therefore essential and of great importance in engineering applications.

L. Dai $(\varangle) \cdot$ X. Wang

Industrial Systems Engineering, University of Regina,

Regina, SK, Canada

e-mail: liming.dai@uregina.ca
Numerous research studies have been conducted in the past by peer researchers and engineers. A thorough review of the publications about sloshing problems prior to 1966 was provided in Absamson and Silverman [1], with focuses on liquid sloshing problems in aerospace. Recently, a detailed review of research works in liquid sloshing was reported by Ibrahim et al. [2]. As per the reviews, liquid sloshing problems were generally investigated experimentally and numerically.

In Berlot [3], for example, the motion of fluid contained in a cylindrical tank driven laterally was analyzed in terms of the combination of surface waves and two dimensional vortexes. Experimentally, Abramson et al. [4] studied the nonlinear lateral sloshing in rigid tanks of various geometries. In their study, the experimental data, primarily on force responses, were presented. The authors found that the primary nonlinear effects observed in all tanks were decreasing with increasing excitation amplitude. Much more experimental research works have been found in the literature and nonlinear responses of the sloshing liquids are evident.

Besides experimental works, liquid sloshing problems were also studied on the basis of computational simulations and numerical analyses advanced by new developments in computers and computational technologies. Volume-of-fluid approach based simulations were carried out by Veldnam et al. [5] to predict the combined liquid/solid body motion. In this work, the stability of numerical coupling between solid-body dynamics and liquid dynamics was analyzed. A numerical scheme was developed by Chen and Price [6] to model the compressible two-fluid flows and simulate liquid sloshing in a partially filled tank. The method proposed in this work demonstrated that the method was suitable to capture free surface waves and to evaluate sloshing pressure loads acting on the tank walls and ceiling. In William 
and Steve [7], a traction control algorithm that operates on the same correlated input signals that a human expert driver would in order to maximize traction is proposed. In these research works, the commonly used dynamic liquid models were developed on the basis of fluid mechanics equations, such as Navier-Stokes equation or that of potential theory. For more accurate results, the equations of motion for flexible container structures have to be coupled with that of the liquid in the container. However, even with super computers, coupling the equations of both liquid and solid can be very computationally demanding. For ordinary design analyses, more practically convenient models are preferred. A combined spherical pendulum and linear pendulum system is developed to produce the same dynamic in-line and crossaxis reaction weight as liquid exhibiting rotary liquid slosh by Kana [8]. Yurchenko and Alevras [9] have designed an $\mathrm{N}$-pendulum, which represents a special case of a physical pendulum. The design of the N-pendulum not only allows uncoupling the natural frequency of the pendulum from its length, but also provides easy control of the frequency and torque.

Dai and Wang [10] studied liquid sloshing in a container with employment of a simplified planar nonlinear pendulum model. Although the study revealed the nonlinear sloshing behavior in a periodically excited tank, the analysis of the study is two dimensional. In fact, though detailed study on three dimensional liquid sloshing is still not available in the literature. Based on the archived documents available to the authors, reliable theoretical or numerical solutions of liquid sloshing in a three-dimensional tank are still in lack. Also, very few research works are found in the literature for studying the effects of continuously varying gravity and effects of liquid viscosity on the liquid motions in the containers subjected to external excitations.

As an alternate approach in studying liquid sloshing in containers, equivalent mechanical models were widely used by the researchers in this field [1]. Spring mass damper models and pendulum models are the two typical mechanical models used for investigating liquid sloshing. The models can be conveniently applied to simulate liquid sloshing and to study the dynamic behaviour of sloshing in containers. Although the two types of model are actually equivalent in terms of mechanical modelling, a threedimensional pendulum model is preferred in this research for the reason that the natural frequency of the pendulum is automatically altered with the changes in pivot acceleration or gravitational acceleration. With a nonlinear conical pendulum model, the three-dimensional liquid sloshing in a carrier is to be studied in this research with considerations of the liquids with or without viscosity and the variations of gravitational gravity acting on the liquids.

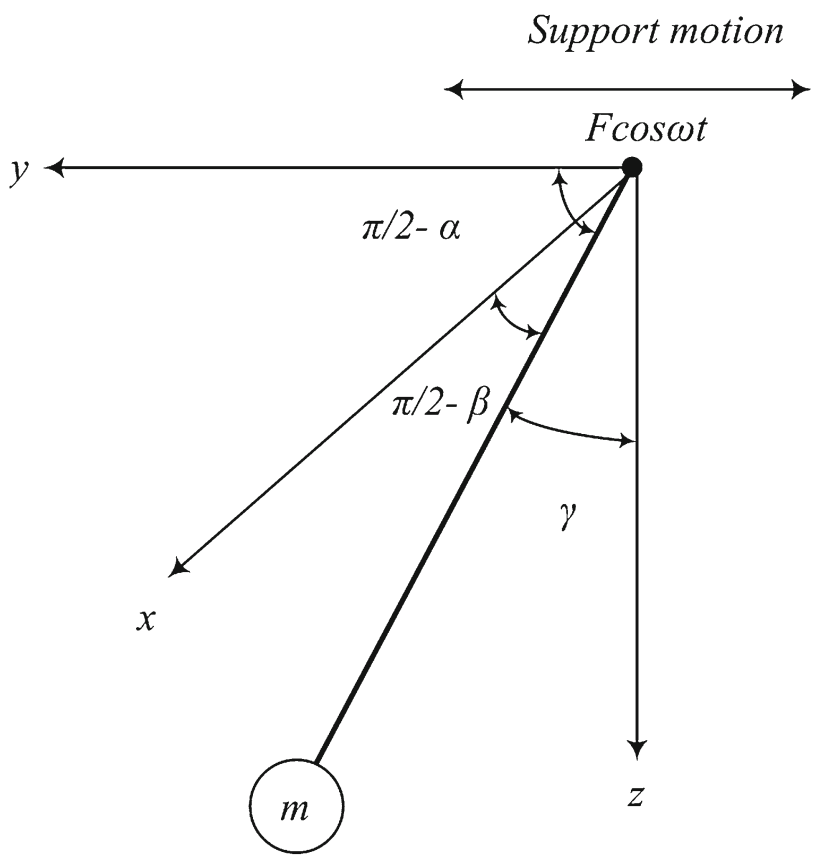

Fig. 1 Conical pendulum model for rotary sloshing

\section{Governing equations and the numerical solutions}

As described in the Introduction, pendulum models are good mechanical analogies for simulating liquid sloshing in containers. One of such pendulum models named the conical pendulum model was described by Abramson and Silverman [1]. This model can be employed for studying the liquid motion in a container of a carrier which may vary its position in space. The advantage of this model is its convenience in describing liquid sloshing in a three-dimensional fashion and the application of external excitations on the pendulum system. The pendulum such established is also convenient for developing governing equations with Newtonian mechanics and reveals the fundamental behavior of the sloshing on a practically sound basis. A sketch of the model is shown in Fig. 1.

As shown in Fig. 1, there are three angles $\alpha, \beta$ and $\gamma$ in the model and the three angles completely determine the position and motion of the pendulum therefore liquid sloshing in the carrier. Among the three angles in the coordinate system, as the length of the pendulum is assumed as a constant, two of them are actually independent and the relationship among the angles can be expressed by the trigonometric function: $\sin ^{2} \alpha+\sin ^{2} \beta=\sin ^{2} \gamma$ for the angles as defined in Fig. 1.

In developing for the governing equations, the following assumptions and conditions are applied:

(1) The container is treated as a rigid object;

(2) The liquid in the container is incompressible and is ideal Newtonian fluid; 


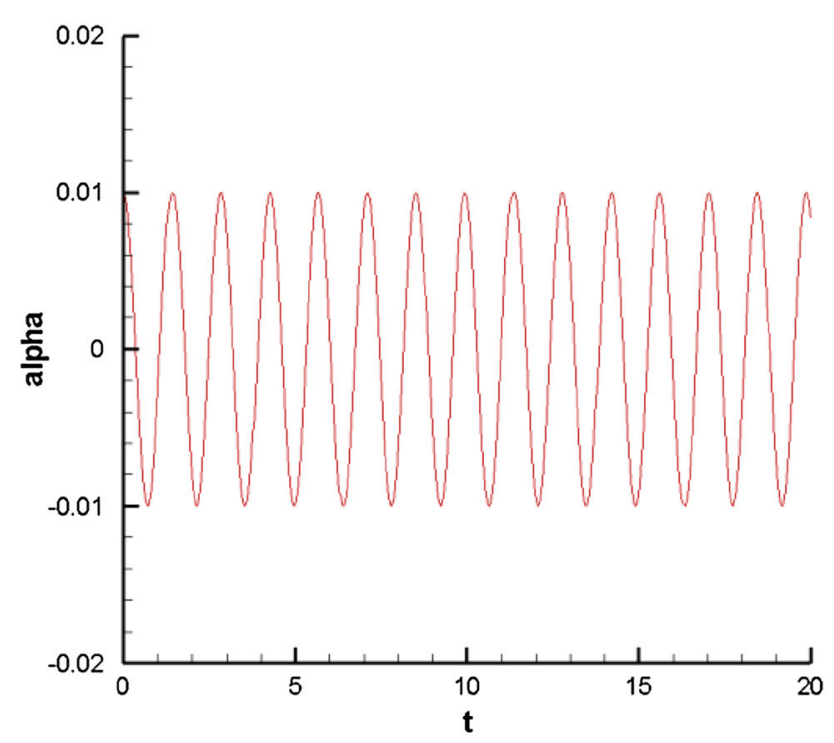

Wave diagram of $\alpha$

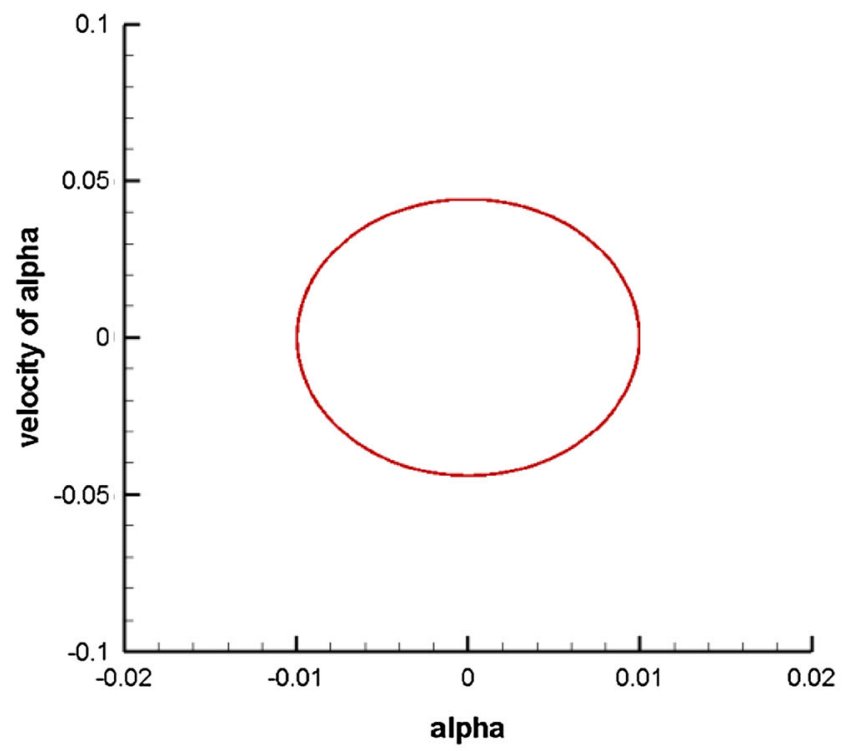

Phase trajectory of $\alpha$

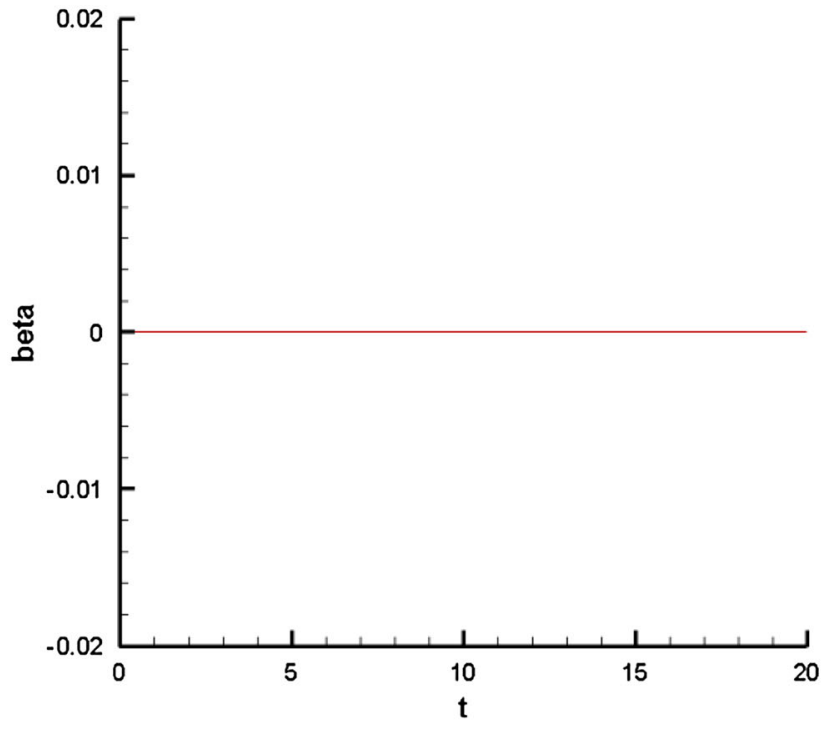

Wave diagram of $\beta$

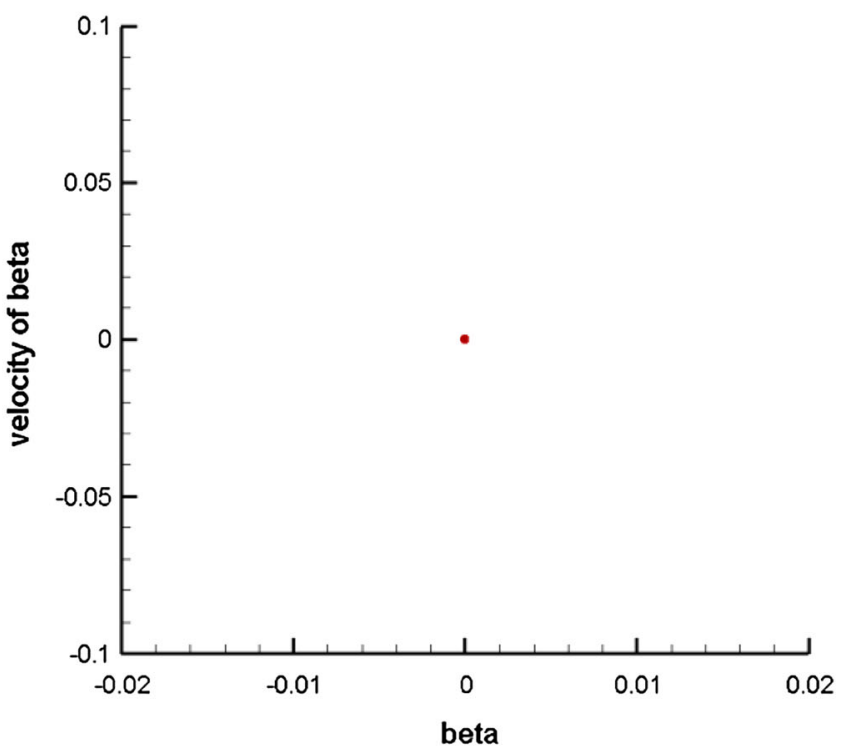

Phase trajectory of $\beta$

Fig. 2 Dynamic behaviors of $\alpha$ and $\beta\left(L=0.5 \mathrm{~m}, h=0.0314 \mathrm{~s}, g=9.8 \mathrm{~m} / \mathrm{s}^{2} F=0.0 \mathrm{~N}, \omega=1.0 \mathrm{rad} / \mathrm{s}, \alpha(0)=0.01 \mathrm{rad}, \dot{\alpha}(0)=0.0 \mathrm{rad} / \mathrm{s}\right.$, $\beta(0)=0.0 \mathrm{rad}, \dot{\beta}(0)=0.0 \mathrm{rad} / \mathrm{s})$

(3) The sloshing amplitude is small such that $\sin \alpha$ can be approximated as $\alpha-\frac{\alpha^{3}}{6}$ and $\cos \alpha \approx 1-\frac{\alpha^{2}}{2}$

(4) The pendulum length relates to the amount of liquid in the container and the amount of liquid does not vary during liquid sloshing;

(5) The external excitation applied on the carrier is periodic and applied in the $y-z$ plane along the $y$ axis.

With the pendulum model such established, the governing equations for the motion of the conical pendulum model can be expressed as [1]:

$$
\frac{d^{2} \alpha}{d t^{2}}+\frac{g}{L} \alpha-\frac{g}{6 L} \alpha^{3}+\frac{1}{2}\left(\frac{d^{2}}{d t^{2}}+\frac{g}{L}\right) \alpha \beta^{2}=F \cos \omega t
$$$$
\frac{d^{2} \beta}{d t^{2}}+\frac{g}{L} \beta-\frac{g}{6 L} \beta^{3}+\frac{1}{2}\left(\frac{d^{2}}{d t^{2}}+\frac{g}{L}\right) \beta \alpha^{2}=0
$$

where $\alpha$ and $\beta$ are the two angular variables, $g$ represents the gravitational acceleration, $L$ denotes the length of the pendulum, $F$ and $\omega$ designate for the amplitude and frequency of the external excitation respectively. As shown in Eq. (1), the two variables are highly coupled for this three-dimensional 


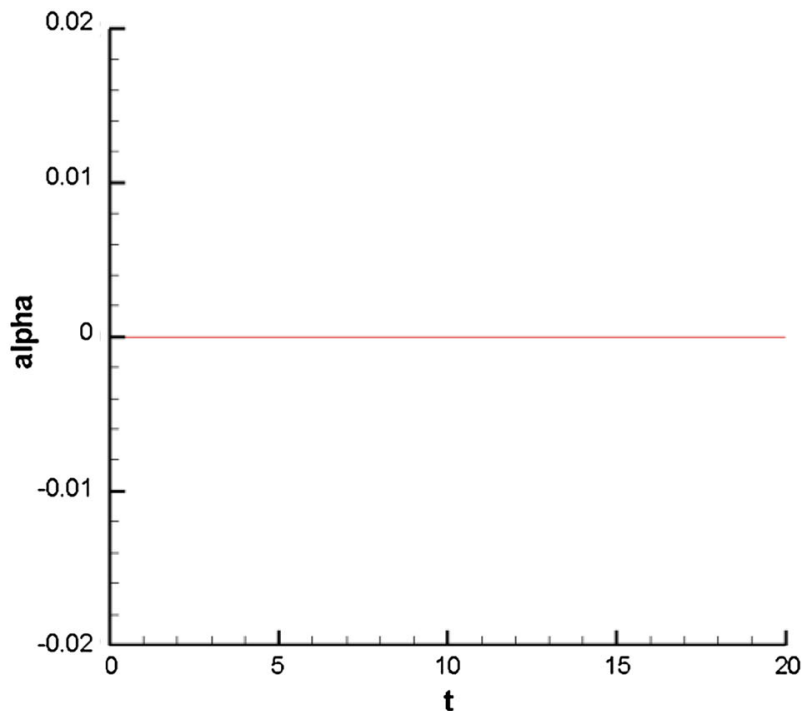

Wave diagram of $\alpha$

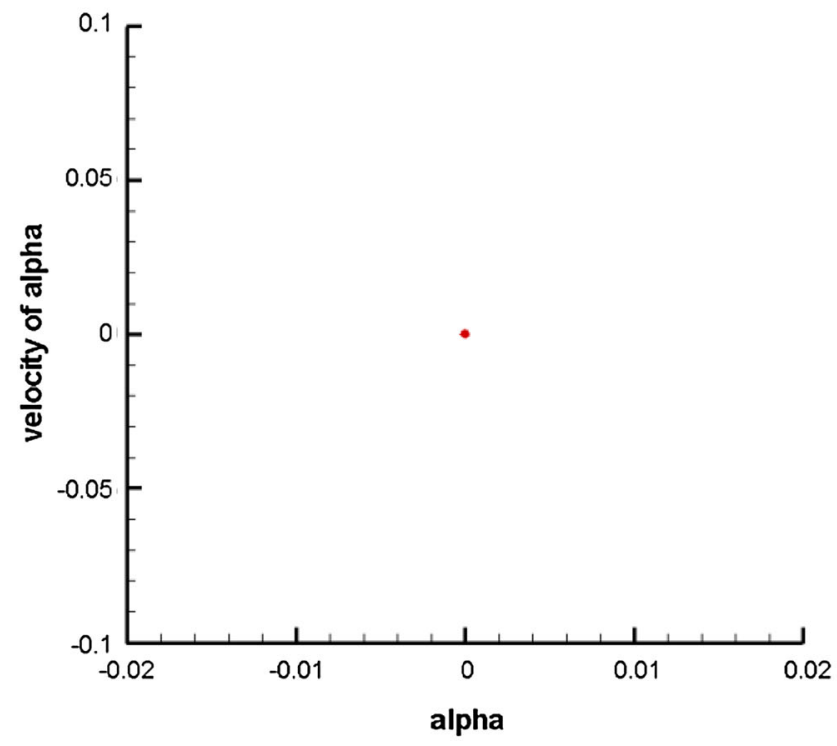

Phase trajectory of $\alpha$

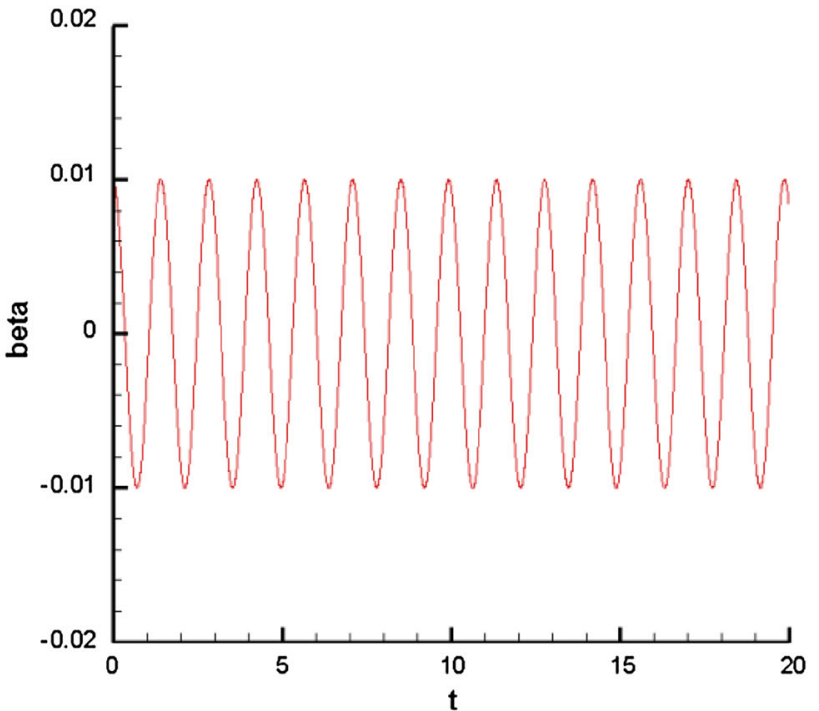

Wave diagram of $\beta$

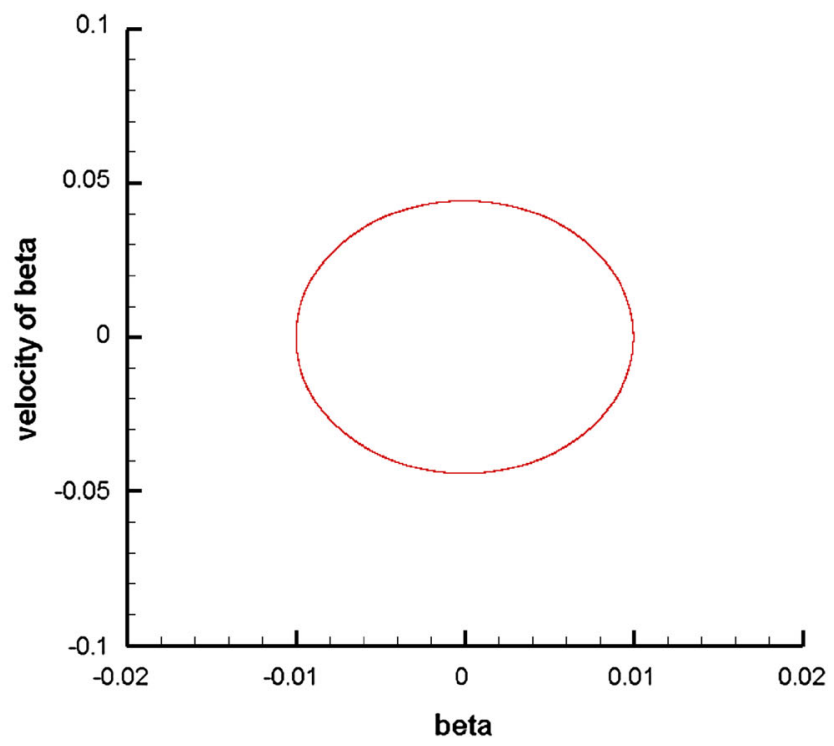

Phase trajectory of $\beta$

Fig. 3 Dynamic behaviors of $\alpha$ and $\beta\left(L=0.5 \mathrm{~m}, h=0.0314 \mathrm{~s}, g=9.8 \mathrm{~m} / \mathrm{s}^{2}, \omega=1.0 \mathrm{rad} / \mathrm{s}, F=0.0 \mathrm{~N}, \alpha(0)=0.00 \mathrm{rad}, \dot{\alpha}(0)=0.0 \mathrm{rad} / \mathrm{s}\right.$, $\beta(0)=0.01 \mathrm{rad}, \dot{\beta}(0)=0.0 \mathrm{rad} / \mathrm{s})$

system. Theoretical solutions for this system are difficult to develop if not impossible, numerical solution is therefore needed. To approach a numerical solution for the system, in this research, the governing equations are decoupled first. To decouple the system, the second order derivative of $\beta$ is described in the following form, as per Eq. (1b).

$$
\begin{aligned}
\ddot{\beta}= & \frac{1}{1+\frac{1}{2} \alpha^{2}}\left(-\frac{g}{L} \beta+\frac{g}{6 L} \beta^{3}-2 \alpha \dot{\alpha} \dot{\beta}-\dot{\alpha}^{2} \beta-\alpha \ddot{\alpha} \beta\right. \\
& \left.-\frac{g}{2 L} \alpha^{2} \beta\right)
\end{aligned}
$$

By plugging Eq. (2) into Eq. (1a) and rearranging the terms, one may have

$$
\begin{aligned}
\ddot{\alpha}= & \frac{1}{1+\frac{1}{2} \beta^{2}-\alpha^{2} \beta^{2} \frac{1}{1+\frac{1}{2} \alpha^{2}}} \times\left(F \cos \omega t-\frac{g}{L} \alpha+\frac{g}{6 L} \alpha^{3}\right. \\
& \left.\quad-\alpha \dot{\beta}^{2}-\frac{\alpha \beta}{1+\frac{1}{2} \alpha^{2}}\left(-\frac{g}{L} \beta+\frac{g}{6 L} \beta^{3}-\beta \dot{\alpha}^{2}\right)\right) \\
& \triangleq F_{\alpha}(\alpha, \dot{\alpha} ; \beta, \dot{\beta})
\end{aligned}
$$

Substitute Eq. (3) into Eq. (1b) to obtain 


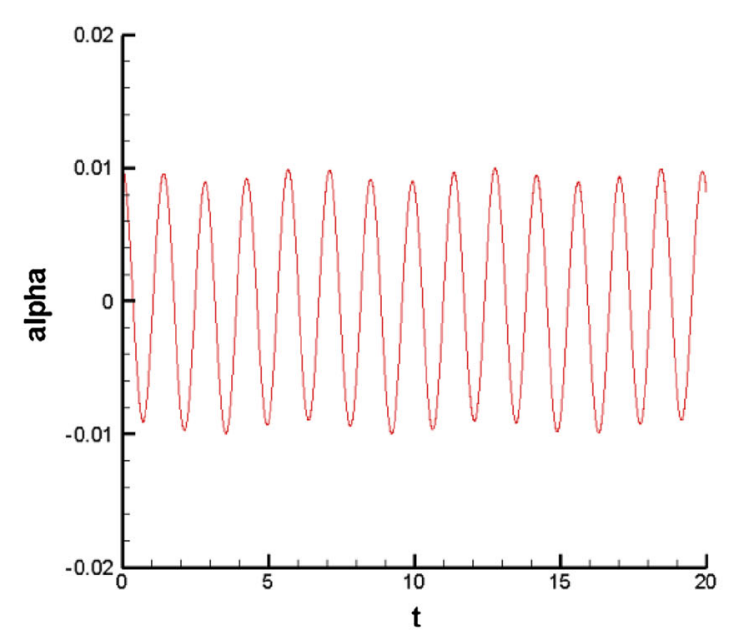

Wave diagram of $\alpha$

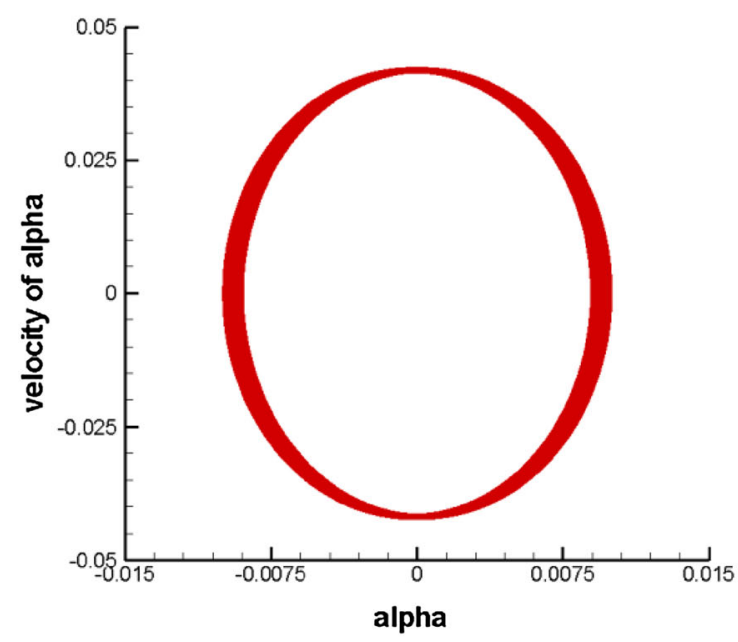

Phase trajectory of $\alpha$

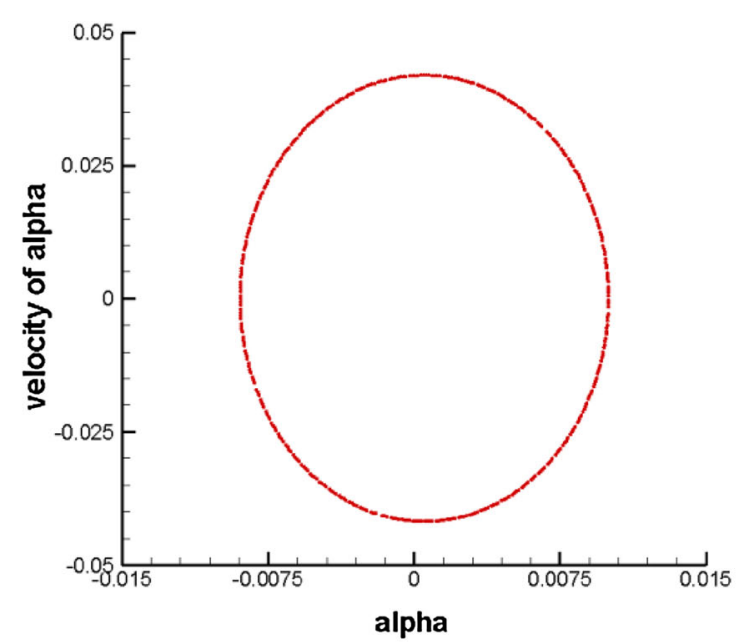

Poincare map of $\alpha$

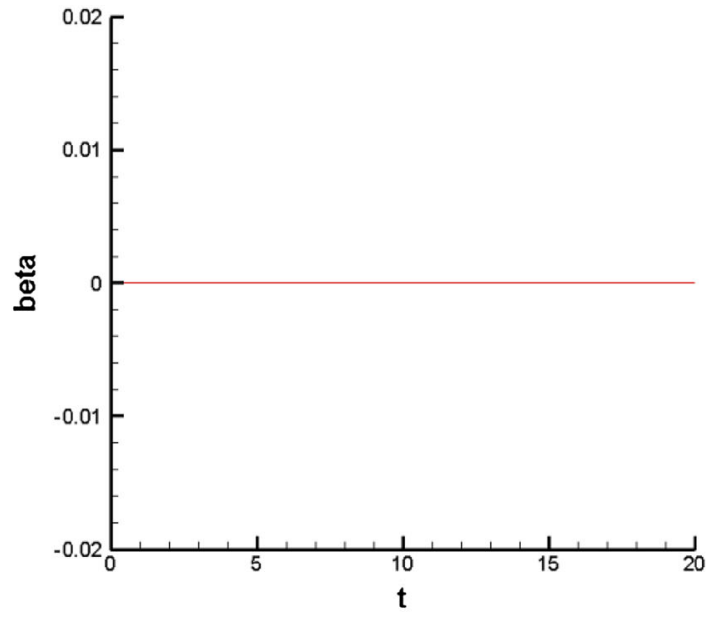

Wave diagram of $\beta$

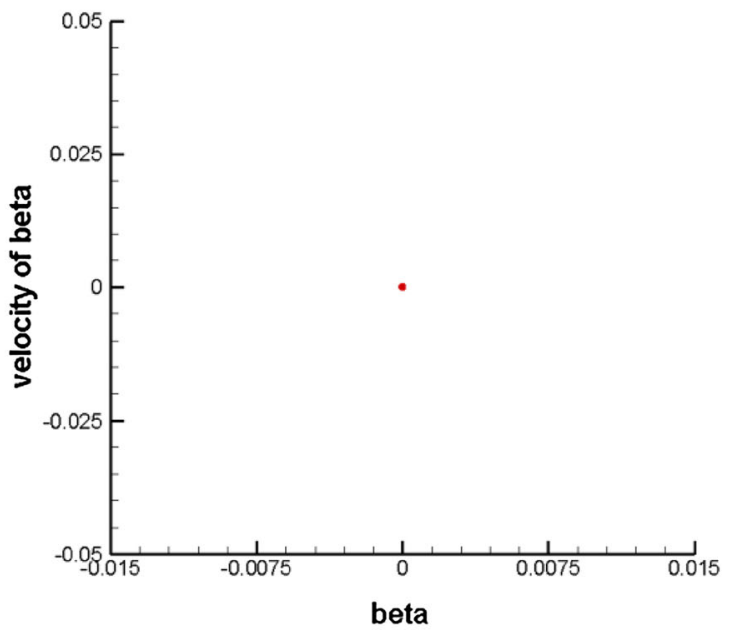

Phase trajectory of $\beta$

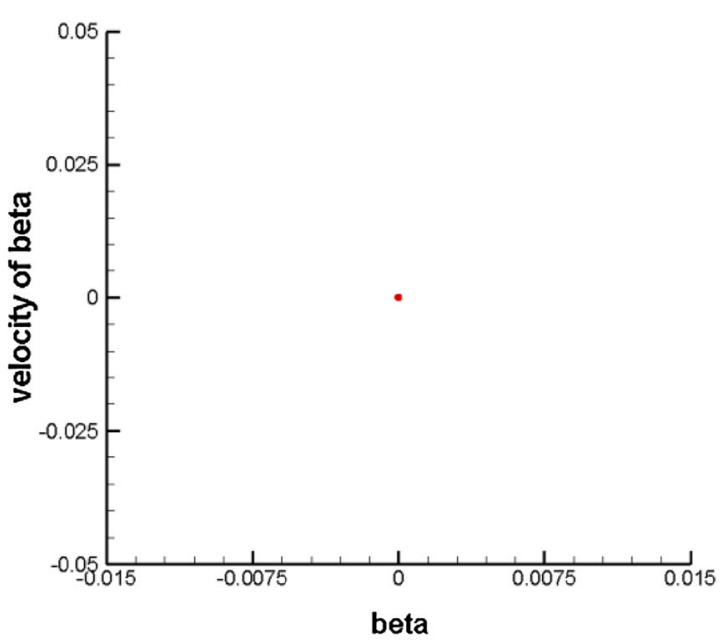

Poincare map of $\beta$

Fig. 4 Dynamic behaviors of $\alpha$ and $\beta\left(L=0.5 \mathrm{~m}, h=0.0314 \mathrm{~s}, g=9.8 \mathrm{~m} / \mathrm{s}^{2}, \omega=1.0 \mathrm{rad} / \mathrm{s}, F=0.01 \mathrm{~N}, \alpha(0)=0.01 \mathrm{rad}, \dot{\alpha}(0)=0.0 \mathrm{rad} / \mathrm{s}\right.$, $\beta(0)=0.0 \mathrm{rad}, \dot{\beta}(0)=0.0 \mathrm{rad} / \mathrm{s})$ 


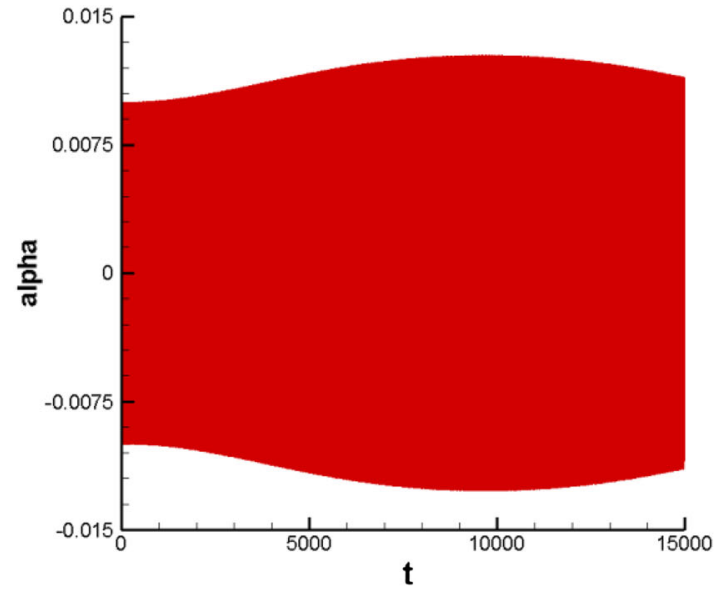

Wave diagram for $\alpha$

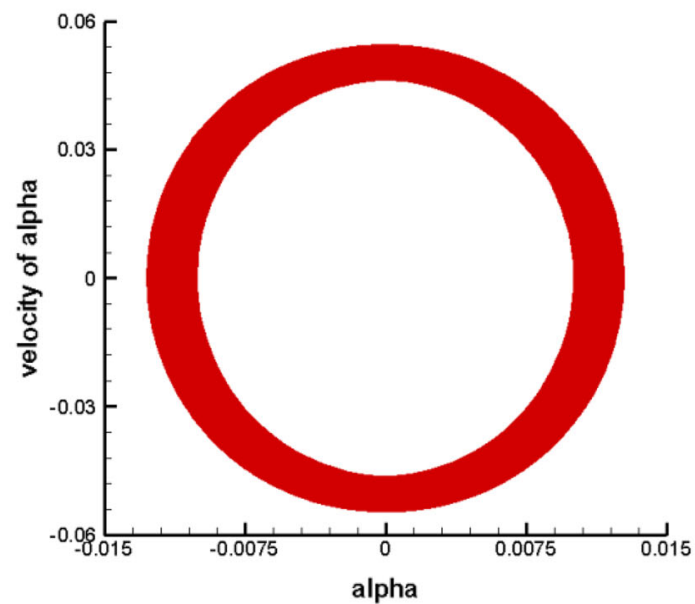

Phase trajectory for $\alpha$

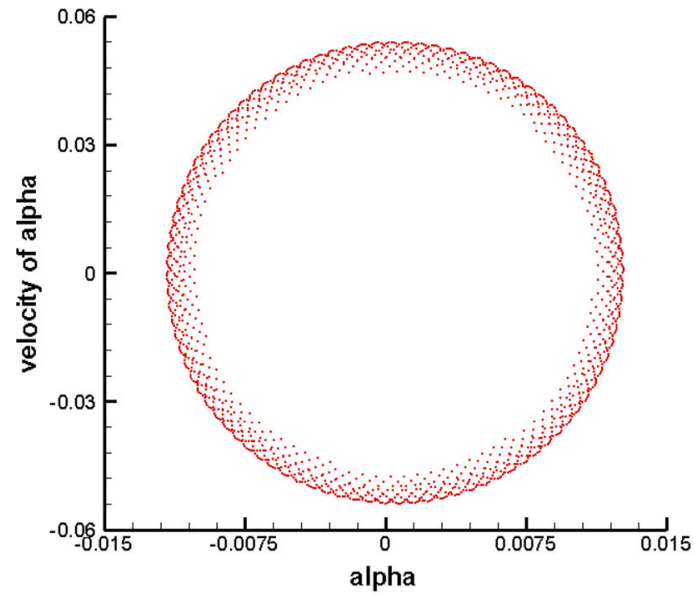

Poincare map of $\alpha$

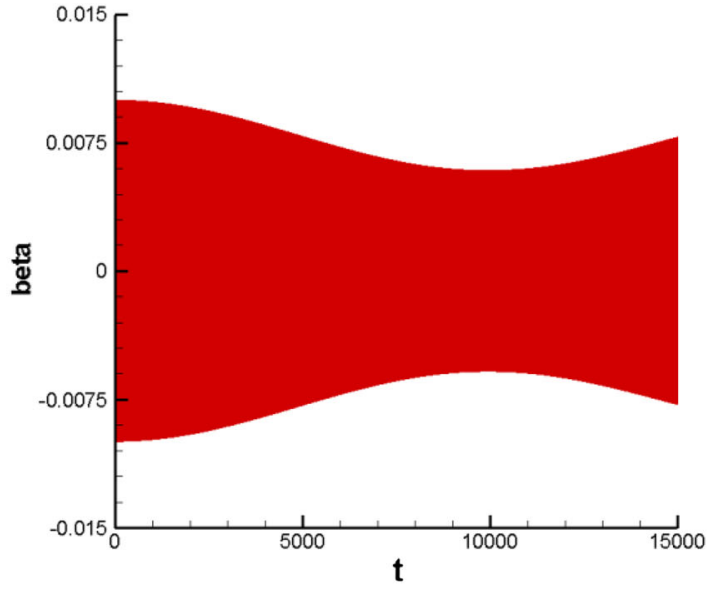

Wave diagram for $\beta$

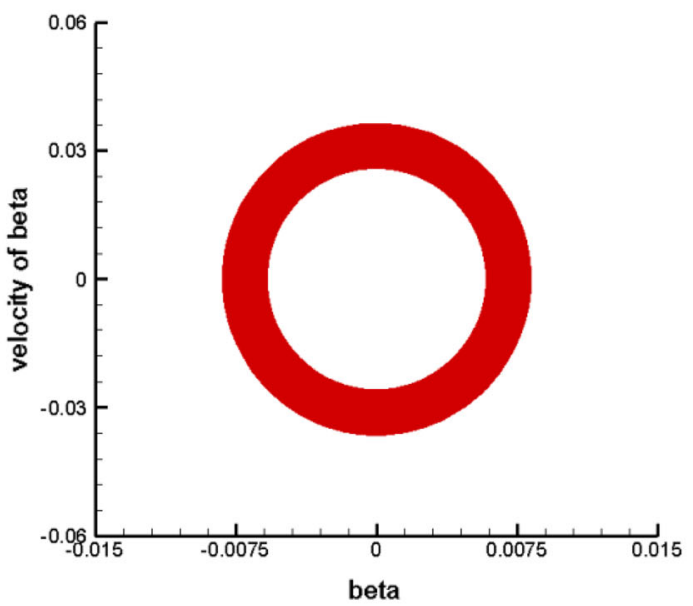

Phase trajectory for $\beta$

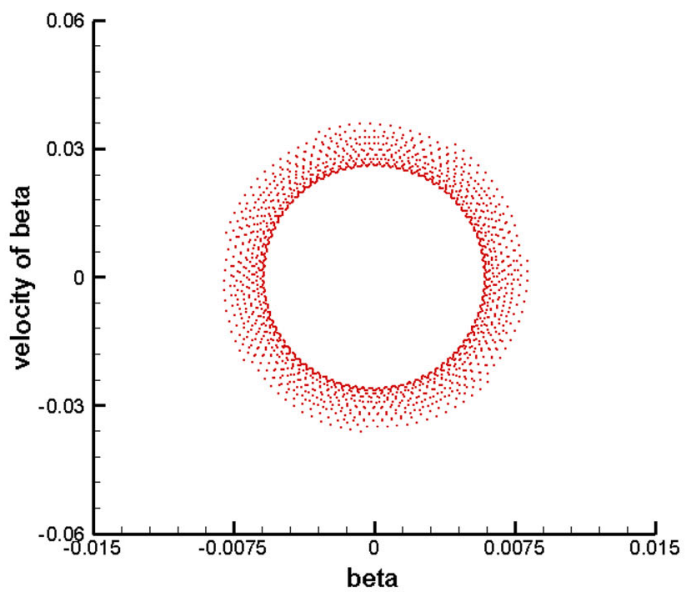

Poincare map of $\beta$

Fig. 5 Dynamic behaviors of the pendulum model $\left(L=0.5 \mathrm{~m}, h=0.01 \mathrm{~s}, g=9.8 \mathrm{~m} / \mathrm{s}^{2}, \omega=1.0 \mathrm{rad} / \mathrm{s}, F=0.01 \mathrm{~N}, \alpha(0)=0.01 \mathrm{rad}\right.$, $\dot{\alpha}(0)=0.0 \mathrm{rad} / \mathrm{s}, \beta(0)=0.0 \mathrm{rad}, \dot{\beta}(0)=0.0 \mathrm{rad} / \mathrm{s})$ 


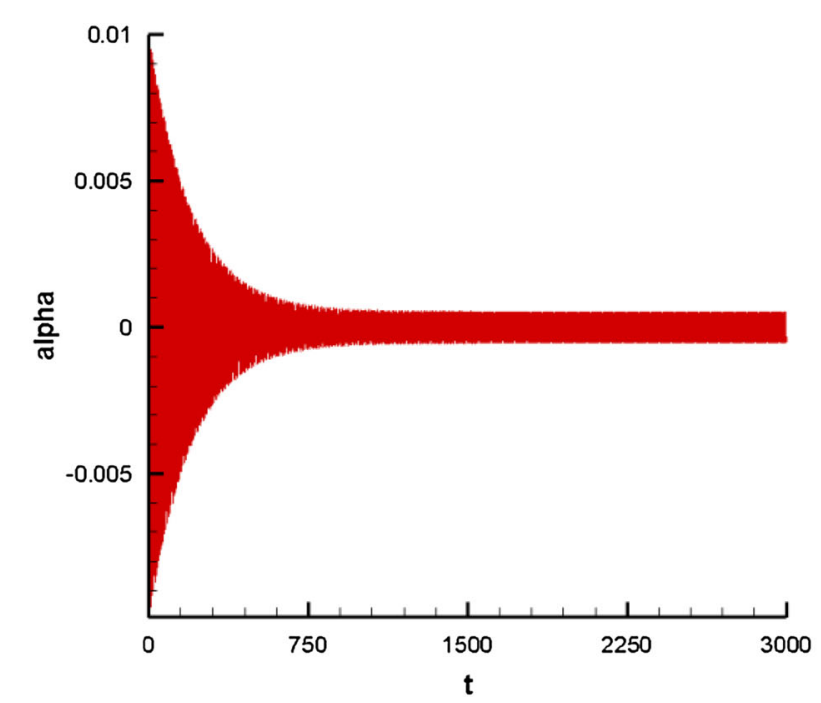

Wave diagram for $\alpha$

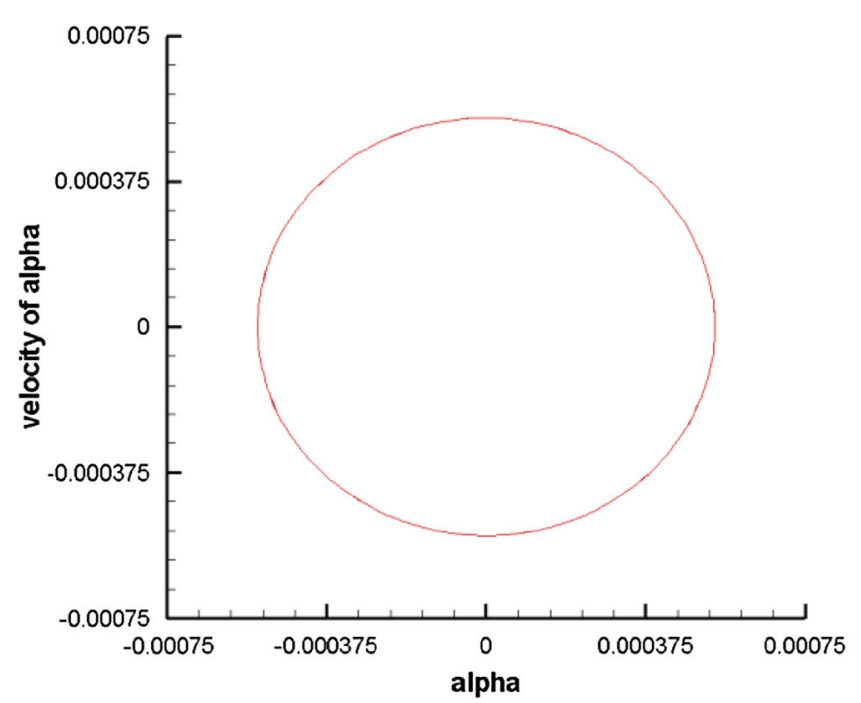

Phase trajectory for $\alpha$

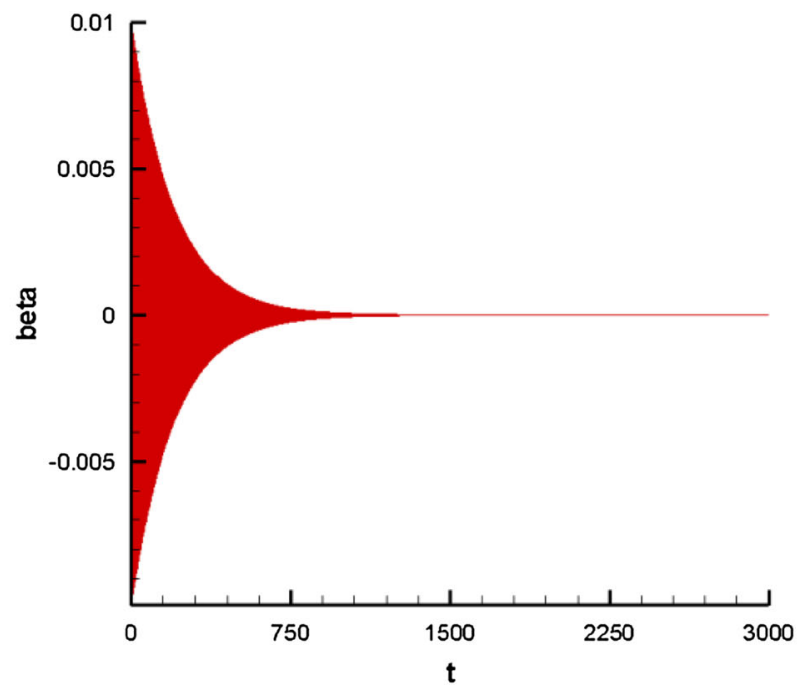

Wave diagram for $\beta$

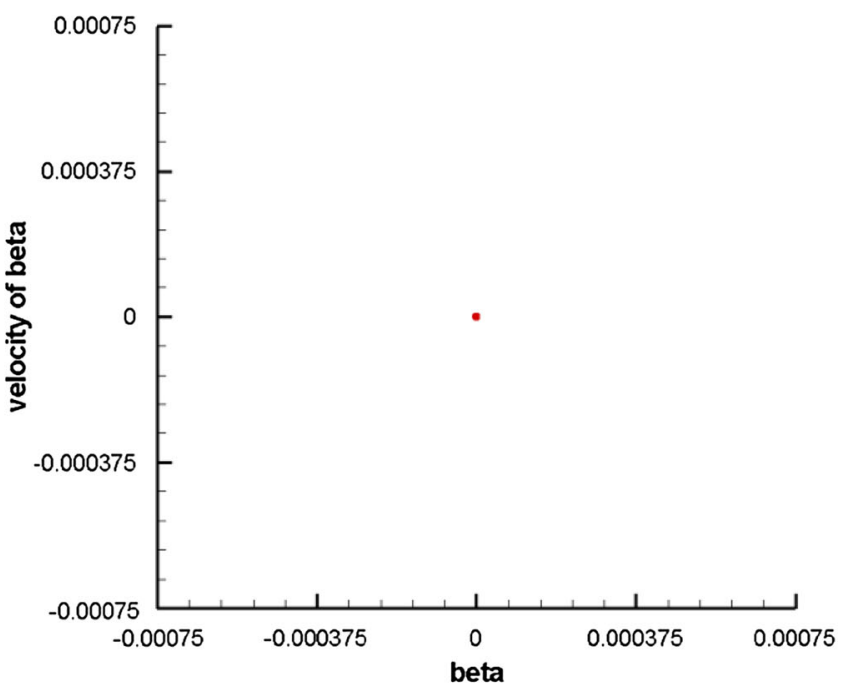

Phase trajectory for $\beta$

Fig. 6 Dynamic behaviors of the pendulum model $\left(L=0.5 \mathrm{~m}, h=0.0314 \mathrm{~s}, g=9.8 \mathrm{~m} / \mathrm{s}^{2}, \omega=1.0 \mathrm{rad} / \mathrm{s}, F=0.01 \mathrm{~N}, C=0.01 \mathrm{~N} \cdot \mathrm{s} / \mathrm{m}\right.$, $\alpha(0)=0.01 \mathrm{rad}, \dot{\alpha}(0)=0.0 \mathrm{rad} / \mathrm{s}, \beta(0)=0.01 \mathrm{rad}, \dot{\beta}(0)=0.0 \mathrm{rad} / \mathrm{s})$

$$
\begin{aligned}
\ddot{\beta}= & \frac{1}{1+\frac{1}{2} \alpha^{2}} \\
& \times\left(-\frac{g}{L} \beta+\frac{g}{6 L} \beta^{3}-\beta\left(\dot{\alpha}^{2}+F_{1}(\alpha, \dot{\alpha} ; \beta, \dot{\beta})\right)\right) \\
& \triangleq F_{\beta}(\alpha, \dot{\alpha} ; \beta, \dot{\beta})
\end{aligned}
$$

To solve the differential equations, the 4th order Runge-Kutta method is considered in this research. By applying the RungeKutta method, the above equations are expressed as four first order differential equations, with the following transformations.

$$
\begin{aligned}
& \alpha=x_{1} \\
& \dot{\alpha}=x_{2}
\end{aligned}
$$

$$
\begin{aligned}
& \beta=x_{3} \\
& \dot{\beta}=x_{4}
\end{aligned}
$$

As such, Eqs. (3) and (4) may take for following forms.

$$
\begin{aligned}
\dot{x}_{1}=x_{2} & \triangleq F_{1}\left(x_{1}, x_{2}, x_{3}, x_{4}\right) \\
\dot{x}_{2}= & \frac{1}{1+\frac{1}{2} x_{3}^{2}-x_{1}^{2} x_{3}^{2} \frac{1}{1+\frac{1}{2} x_{1}^{2}}} \times\left\{F \cos \omega t-c x_{2}-\frac{g}{L} x_{1}\right. \\
& +\frac{g}{6 L} x_{1}^{3}-2 x_{2} x_{3} x_{4}-x_{1} x_{4}^{2}-\frac{g}{2 L} x_{1} x_{3}^{2} \\
& -\frac{x_{1} x_{3}}{1+\frac{1}{2} x_{1}^{2}}\left(-c x_{4}-\frac{g}{L} x_{3}+\frac{g}{6 L} x_{3}^{3}-2 x_{1} x_{2} x_{4}\right. \\
& \left.\left.-x_{3} x_{2}^{2}-\frac{g}{2 L} x_{1}^{2} x_{3}\right)\right\}
\end{aligned}
$$




$$
\begin{aligned}
& \triangleq \\
\dot{x}_{3}=F_{2}\left(x_{1}, x_{2}, x_{3}, x_{4}\right) & \triangleq F_{3}\left(x_{1}, x_{2}, x_{3}, x_{4}\right) \\
\dot{x}_{4}= & \frac{1}{1+\frac{1}{2} x_{1}^{2}} \times\left(-c x_{4}-\frac{g}{L} x_{3}+\frac{g}{6 L} x_{3}^{3}-2 x_{1} x_{2} x_{4}\right. \\
& \left.-x_{3}\left(x_{2}^{2}+x_{1} F_{2}\left(x_{1}, x_{2}, x_{3}, x_{4}\right)\right)-\frac{g}{2} x_{1}^{2} x_{3}\right) \\
\triangleq & F_{4}\left(x_{1}, x_{2}, x_{3}, x_{4}\right)
\end{aligned}
$$

Equation (6) can be expressed with vector form

$\dot{\vec{X}}=\vec{f}(t, \vec{X})$,

where

$\vec{X}=\left(x_{1}, x_{2}, x_{3}, x_{4}\right)^{T}$

$\vec{f}(t, \vec{X})=\left(F_{1}, F_{2}, F_{3}, F_{4}\right)^{T}$

The solutions of Eq. (7) can be given by

$\vec{X}_{n+1}=\vec{X}_{n}+\frac{1}{6} h\left(\vec{K}_{1}+\vec{K}_{2}+\vec{K}_{3}+\vec{K}_{4}\right)$

where $h$ represents the time step, and the vectors $\vec{K}_{i}(i=$ 1-4) can be calculated by:

$\vec{K}_{1}=\vec{f}\left(t_{n}, \vec{X}_{n}\right)$

$\vec{K}_{2}=\vec{f}\left(t_{n}+\frac{1}{2} h, \vec{X}_{n}+\frac{1}{2} h \vec{K}_{1}\right)$

$\vec{K}_{3}=\vec{f}\left(t_{n}+\frac{1}{2} h, \vec{X}_{n}+\frac{1}{2} h \vec{K}_{2}\right)$

$\vec{K}_{4}=\vec{f}\left(t_{n}+h, \vec{X}_{n}+h \vec{K}_{3}\right)$

\section{Numerical results and discussions}

\subsection{Evaluation of the model and numerical results}

By applying the approach described above, the numerical results of the 3-D liquid sloshing represented by the pendulum model can be obtained. To ensure the correctness and validation of the approach described in the previous section, the numerical results of some specific cases are evaluated with the model, of which the physical phenomena of liquid sloshing are known in general. A case with initial conditions of $\alpha(0)=0.01 \mathrm{rad}, \alpha^{\prime \prime}$ and $\beta(0)=0.0 \mathrm{rad}, \beta^{\prime \prime}$ are considered first. When no external excitation is applied, i.e. $\mathrm{F}=0.0$, the motion of the pendulum should be a free oscillation. If inviscid liquid is further assumed, or no damping on the pendulum system, a perfect harmonic oscillatory motion of the pendulum can be expected. With these considerations and assumptions, numerical calculations are performed with the results plotted in Fig. 2.

As can be seen from Fig. 2, as expected, motion of the pendulum is merely in the $\alpha$-plane and no motion is seen in

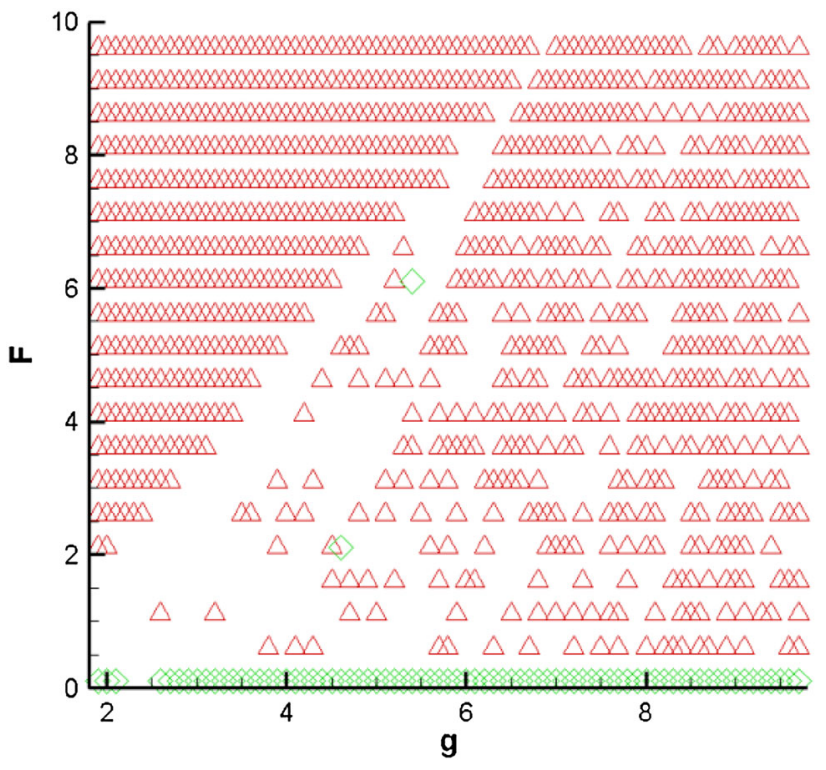

Fig. 7 Region diagrams with respect to $\alpha(L=0.5 \mathrm{~m}, h=0.01 \mathrm{~s}, \omega=$ $1.0 \mathrm{rad} / \mathrm{s}, C=1.0 \times 10^{-7} \mathrm{~N} \cdot \mathrm{s} / \mathrm{m}, \alpha(0)=0.01 \mathrm{rad}, \dot{\alpha}(0)=0.0 \mathrm{rad} / \mathrm{s}$, $\beta(0)=0.01 \mathrm{rad}, \dot{\beta}(0)=0.0 \mathrm{rad} / \mathrm{s})$

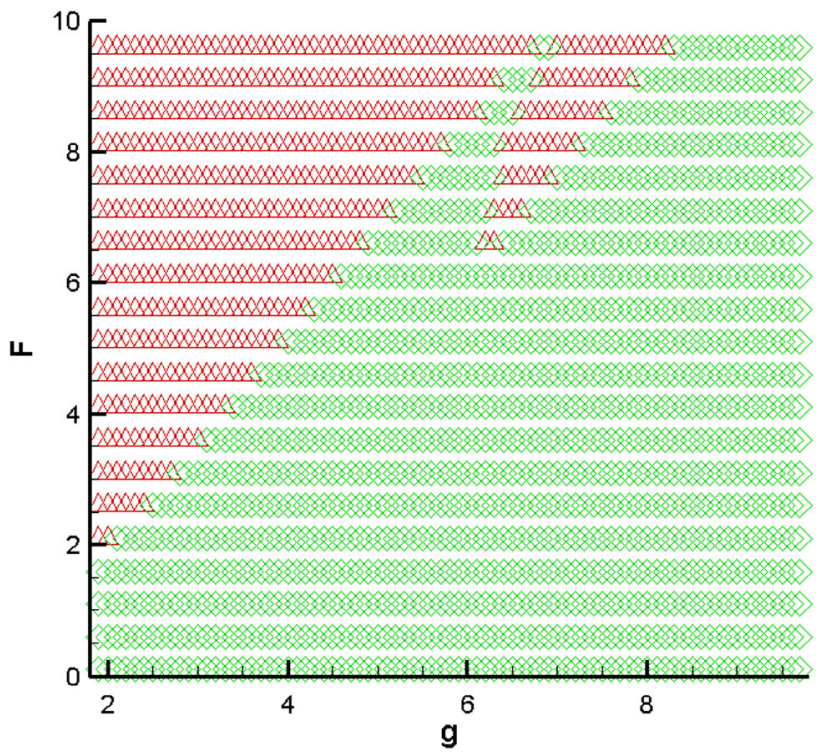

Fig. 8 Region diagrams with respect to $\alpha(L=0.5 \mathrm{~m}, h=0.01 \mathrm{~s}, \omega=$ $1.0 \mathrm{rad} / \mathrm{s}, C=1.0 \times 10^{-2} \mathrm{~N} \cdot \mathrm{s} / \mathrm{m}, \alpha(0)=0.01 \mathrm{rad}, \dot{\alpha}(0)=0.0 \mathrm{rad} / \mathrm{s}$, $\beta(0)=0.01 \mathrm{rad}, \dot{\beta}(0)=0.0 \mathrm{rad} / \mathrm{s})$

the $\beta$-plane. Also, the oscillation of the pendulum is indeed a perfect harmonic periodic motion, as can be clearly seen from the wave diagram and phase trajectory obtained.

When the initial conditions are changed to $\alpha(0=$ $0.0 \mathrm{rad}, \alpha^{\prime \prime}$ and $\beta(0)=0.01, \beta^{\prime \prime}$, oscillation of the pendulum may only be seen in the $\beta$-plane, and the corresponding oscillation can only be harmonic, as shown in the figures of Fig. 3. 


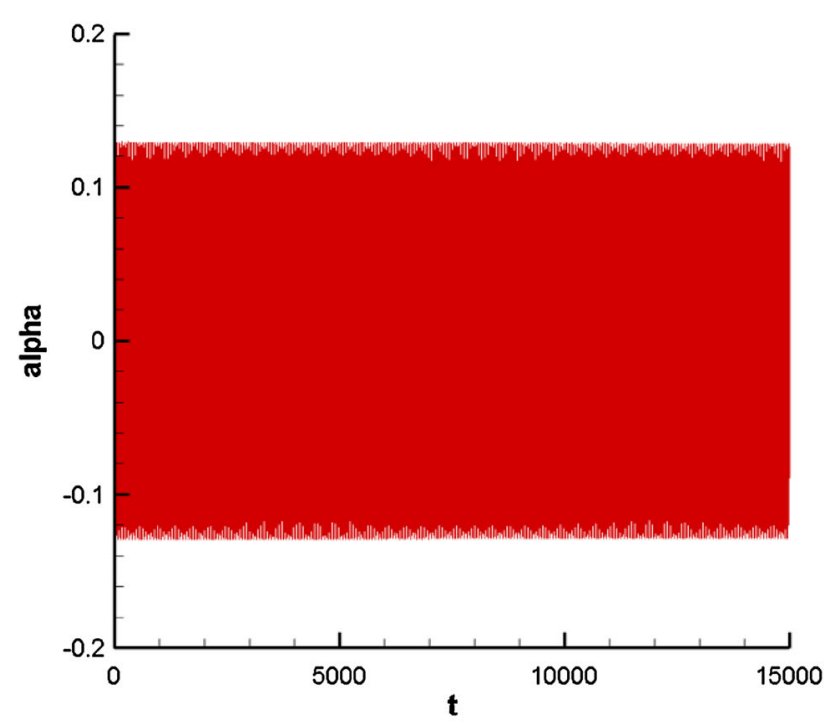

Wave diagram

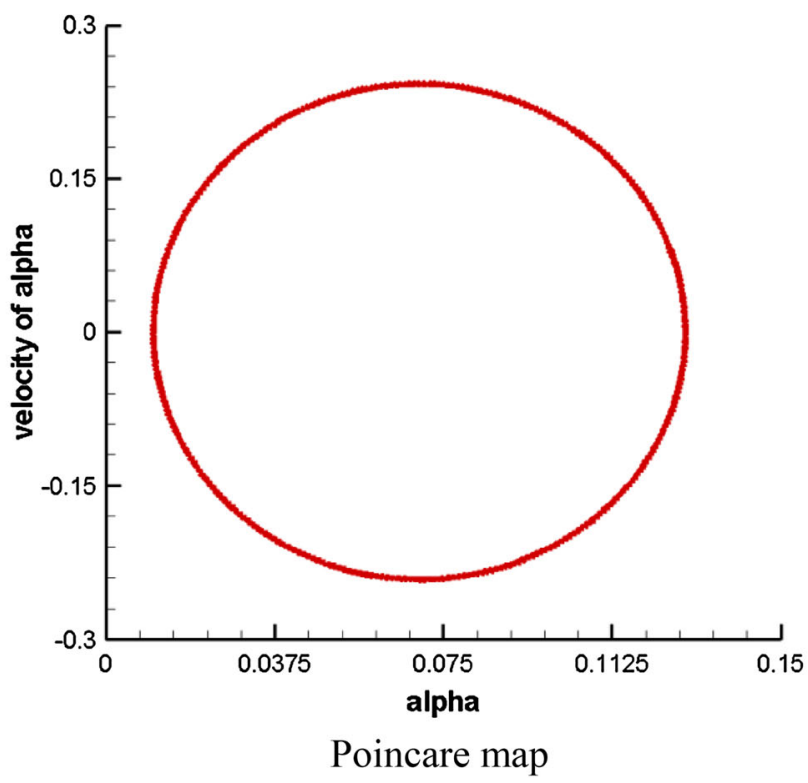

Fig. 9 Dynamic behaviors of the pendulume model $(F=1.1 \mathrm{~N}, g=$ $8.4 \mathrm{~m} / \mathrm{s}^{2}, L=0.5 \mathrm{~m}, h=0.01 \mathrm{~s}, \omega=1.0 \mathrm{rad} / \mathrm{s}, C=1.0 \times 10^{-7} \mathrm{~N} \cdot \mathrm{s} / \mathrm{m}$, $\alpha(0)=0.01 \mathrm{rad}, \dot{\alpha}(0)=0.0 \mathrm{rad} / \mathrm{s}, \beta(0)=0.01 \mathrm{rad}, \dot{\beta}(0)=$ $0.0 \mathrm{rad} / \mathrm{s})$

When an external excitation of amplitude $0.01 \mathrm{~N}$ is applied to the first case, the numerical results obtained show the responses as illustrated in Fig. 4. Although there is no motion in the $\beta$-plane as expected, the motion in the $\alpha$-plane is quasiperiodic. This result agrees with that reported by Dai and Wang [10].

From the results obtained by employing the model presented with the numerical solutions, one may conclude that the results generated with the model and associated numerical approach are reasonable and in good agreement with the fundamental concepts of dynamics.

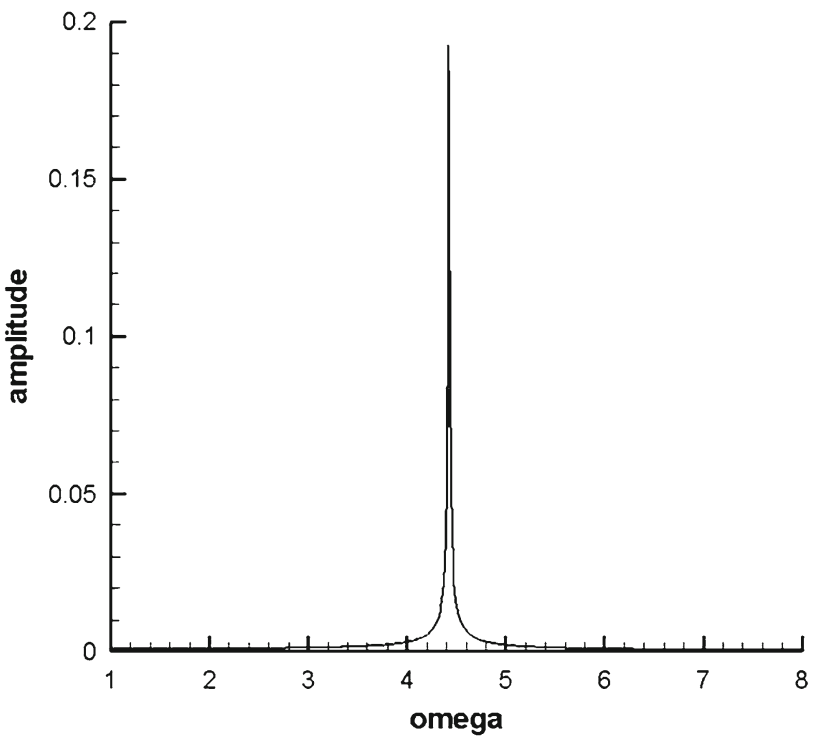

Fig. 10 The amplitude under different external frequencies with respect to $\alpha\left(L=0.5 \mathrm{~m}, g=9.8 \mathrm{~m} / \mathrm{s}^{2}, F=0.01 \mathrm{~N}, C=0.01 \mathrm{~N}\right.$. $\mathrm{m} / \mathrm{s}, d t=0.0314 \mathrm{~s}, \alpha(0)=0.01 \mathrm{rad}, \dot{\alpha}(0)=0.0 \mathrm{rad} / \mathrm{s})$

\subsection{3-D sloshing of inviscid liquid with external excitation}

The sloshing of the liquid in a general three dimension case is considered first. As described previously, the angles $\alpha$ and $\beta$ are independent for determining the oscillations of the pendulum. With the model presented and initial displacements in the $\alpha$ and $\beta$ planes, the sloshing of the liquid in three dimensional space is determined with the model presented. The typical responses of the pendulum are shown in Fig. 5. In this case, the response is non-periodic or quasiperiodic with some regularity.

One may notice, from Fig. 4, non-periodic responses may also occur even if no initial displacement is presented in $\beta$-plane. As can be seen from Fig. 5, the responses of the pendulum is more complex with non-periodic oscillations in both the $\alpha$ and $\beta$ planes. It is interesting to notice that the amplitudes of the oscillations are varying with time in the fashion that $\alpha$ increases while $\beta$ decreases and $\alpha$ decreases as $\beta$ increases. Although the oscillations show regularities in both the $\alpha$ and $\beta$ planes, they are not perfectly periodic. With inviscid liquid, the oscillations in both the planes are found quasiperiodic for most of the parameters and conditions considered in this research, when the sloshing is stabilized.

\subsection{3-D sloshing of viscous liquid}

The viscosity of liquid usually plays an inconspicuous even ignorable role on the sloshing motion when the sloshing amplitude is very small. In the cases that the sloshing becomes larger or complex, the existence of liquid viscosity is considered to have noticeable influence on the overall liquid sloshing. To demonstrate the effects of liquid viscosity 

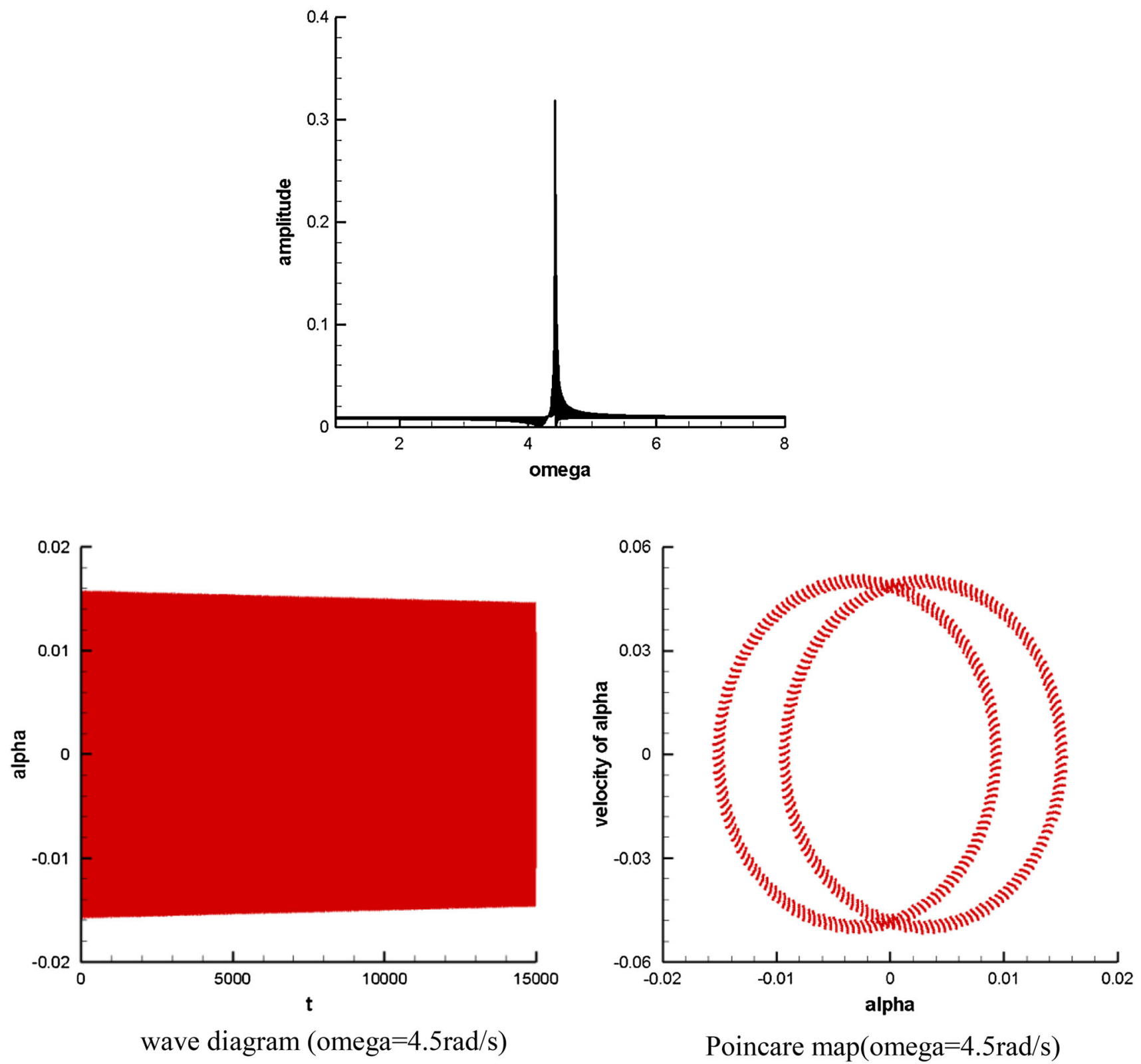

Fig. 11 The amplitude under different external frequencies with respect to $\alpha\left(L=0.5 \mathrm{~m}, g=9.8 \mathrm{~m} / \mathrm{s}^{2}, F=0.01 \mathrm{~N}, C=0.00001 \mathrm{~N} \cdot \mathrm{m} / \mathrm{s}\right.$, $d t=0.0314 \mathrm{~s}, \alpha(0)=0.01 \mathrm{rad}, \dot{\alpha}(0)=0.0 \mathrm{rad} / \mathrm{s})$

on the liquid motion, the sloshing of viscous liquid with the other parameters identical to that shown in Fig. 5 is evaluated, with the results shown in Fig. 6.

It can be observed from Fig. 6, when the viscosity of liquid is considered (even it is very small), the motion of $\beta$ is weakened and eventually vanished. Whereas the motion in the $\alpha$-plane eventually becomes perfectly periodic with uniform amplitude when the motion is stabilized. This implies that liquid sloshing is finally reduced to a 2-D liquid motion in the $\alpha$-plane, for viscous liquid.

To systematically study the responses of the liquid in the carrier considered, it will be ideal to examine the responses corresponding to various parameters over a large range. A single valued index named the periodicity ratio (PR) was introduced for diagnosing the characteristics of nonlinear systems [11,12]. The PR index can be conveniently used as a criterion to diagnose chaotic, periodic or quasiperiodic motions of a nonlinear system, and more significantly, to quantify the nonlinear behavior in between chaos and periodicity of a dynamic system without plotting any phase diagrams or Poincare maps. With employment of the PR index, the regular-irregular region diagrams can be plotted to demonstrate the properties of sloshing with varying system parameters and conditions. Such a region diagram is 


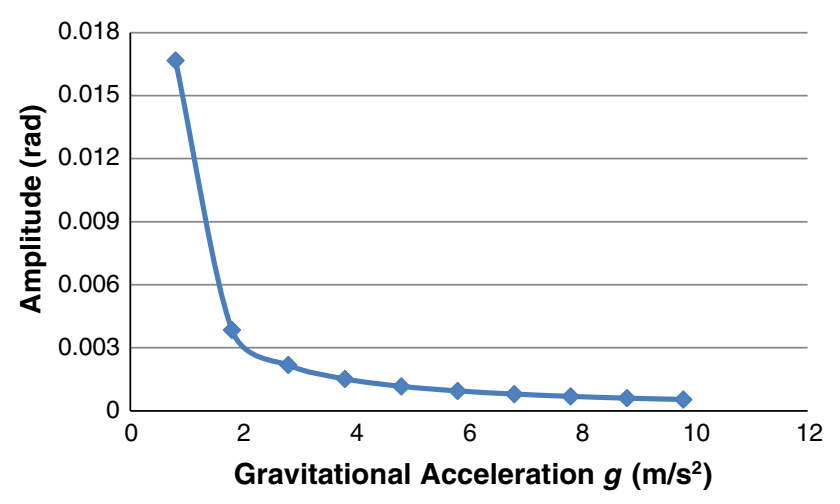

Fig. 12 The influence of gravitational acceleration on the sloshing amplitude $(F=0.01 \mathrm{~N}, C=0.01 \mathrm{~N} \cdot \mathrm{m} / \mathrm{s}, \omega=1.0 \mathrm{rad} / \mathrm{s}, \mathrm{L}=0.5 \mathrm{~m}$, $h=0.01 \mathrm{~s}, \alpha(0)=0.01 \mathrm{rad}, \dot{\alpha}(0)=0.0, \beta(0)=0.01 \mathrm{rad}, \beta(0)$ $=0.0)$

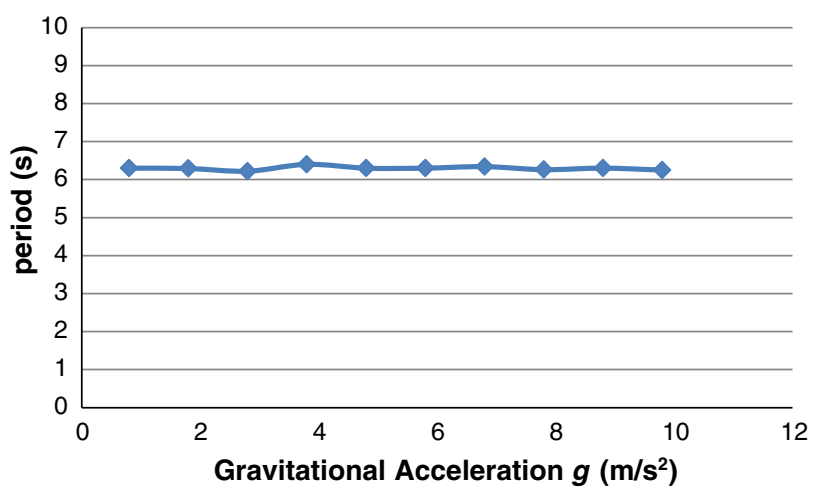

Fig. 13 The influence of gravitational acceleration on the sloshing amplitude $(F=0.01 \mathrm{~N}, C=0.01 \mathrm{~N} \cdot \mathrm{m} / \mathrm{s}, \omega=1.0 \mathrm{rad} / \mathrm{s}, L=0.5 \mathrm{~m}$, $h=0.01 \mathrm{~s}, \alpha(0)=0.01 \mathrm{rad}, \dot{\alpha}(0)=0.0, \beta(0)=0.01 \mathrm{rad}, \beta(0)=0.0)$

shown in Fig. 7, for which the liquid viscosity is small and the ranges of excitation amplitude $F$ and gravitational acceleration $g$ are $0.0-10.0$ and $1.8-9.8$ respectively. In the diagram, the red triangles represent irregular motion while the green diamonds denote periodic motion of the liquid. The typical irregular $(\mathrm{PR}=0)$ and regular $(\mathrm{PR}=1)$ liquid motions are as that shown in Figs. 5 and 6 respectively. The blank areas in the diagram represent the non-periodic cases neither perfect regular nor perfect irregular.

As can be seen from Fig. 7, most responses of the liquid are irregular and non-periodic, only a small area at the bottom of the diagram covers regular or periodic responses. When $F$ is increased, the responses of the liquid are irregular. Further increase of $F$ will lead to divergence representing rotation of the pendulum that is out of the scope of this research.

Liquid viscosity may have significant effects on the sloshing corresponding to the given system parameters. When viscosity of the liquid becomes larger, the energy of the pendulum is reduced and its motion tends to be periodic in the $\alpha$-plane. Indeed, as shown in Fig. 8, most areas in the regularirregular region diagram become regular or periodic when the viscosity is increased from $c=1.0 \times 10^{-7} \mathrm{~N} \cdot \mathrm{s} / \mathrm{m}$ to $c=1.0 \times 10^{-2} \mathrm{~N} \cdot \mathrm{s} / \mathrm{m}$. A typical irregular response in this case is graphically shown in Fig. 9 which shows quasiperiodic response of the system.

It is interesting to notice, in the research, resonance may occur for this nonlinear system. Figure 10 shows a resonance case for a larger viscous liquid. When the viscosity of the liquid is smaller, as shown in Fig. 11, the amplitude of the sloshing becomes greater and response of liquid sloshing is more complex, in comparison with that of Fig. 10. The liquid motion in this case is shown in wave form and Poincare map of Fig. 11. This is a more complex quasiperiodic case with the two loops formed by small curves.

\subsection{Influence of gravitational acceleration}

In carriers moving in space, such as missiles or rockets, the gravitational acceleration varies. The influence of gravitational acceleration on the motion of the carriers is significant. With the model of the research, the analysis of the gravitational effects on the liquid motion becomes readily available.

Varying gravitational acceleration may cause regular or irregular sloshing of the liquid in a carrier, as shown in the region diagrams of Figs. 7 and 8. The relationship between the sloshing amplitude and the gravitational acceleration is shown in Fig. 12. Obviously, the relstionship is nonlinear and the amplitude may become very large when the gravity is small. As can be seen in the figure, the sloshing amplitude increases dramatically when the gravitational acceleration in reduced to a certain value (about 2 in the case shown). This implies the sloshing may become volatile and cause negative influence to the motion and safety of the carriers, when the carriers reach a high level. When the gravity is small enough, the pendulum may rotate with a constant speed. However, this is not the research interest of this study.

Although the sloshing amplitude is noticeably affected by the gravitational acceleration, the effect of varying gravitational acceleration on the frequency of liquid sloshing is relatively small. As shown in Fig. 13, the period of oscillation remains almost the same for the gravitational acceleration varying from 0.8 to 9.8 .

\section{Conclusion}

This research investigates the effects of liquid viscosity and gravity on the three dimensional nonlinear sloshing of the liquid in a carrier subjected to periodic excitations. Such an investigation is not seen in the literature. When the amplitude of the external excitation is properly low, the sloshing in the carrier can be either periodic or quasiperiodic. The sloshing can be stabilized and reduced to periodic when the liquid viscosity is large enough. Resonances of liquid sloshing are found under certain conditions. The amplitude of the 
sloshing is nonlinearly increasing with the reduction of the gravitational acceleration. The liquid sloshing can be unstable and volatile if the gravitational acceleration reaches certain low value. However, the effect of varying gravitational acceleration on the frequency of the sloshing is insignificant as per the research results. The findings of the research are significant to research on liquid sloshing in a carrier and the design of aerospace vehicles carrying liquid cargo or liquid fuel.

\section{References}

1. Abramson HN, Silverman S (1966) The dynamic behavior of liquids in moving containers. NASA SP-106, Washington

2. Ibrahim RA, Pilipchuk VN, Ikeda T (2001) Recent advances in liquid sloshing dynamics. Appl Mech Rev 54(2):133-199

3. Berlot RR (1959) Production of rotation in a confined liquid through translational motion of the boundaries. J Appl Mech 26:513-516

4. Abramson HN, Chu WH, Kana DD (1966) Some studies of nonlinear lateral sloshing in rigid containers. J Appl Mech 33(4):777-784
5. Veldman AEP, Gerrits J, Luppes R et al (2007) The numerical simulation of liquid sloshing on board spacecraft. J Comput Phys 224:82-99

6. Chen YG, Price WG (2009) Numerical simulation of liquid sloshing in a partially filled container with inclusion of compressibility effects. Phys Fluids 21:112105

7. William T, Steve C (2013) Adaptive vehicle traction control: combined longitudinal and lateral motion. Int J Dyn Control 1(3):239253

8. Kana DD (1989) Validated spherical pendulum model for rotary slosh. AIAA J Spacecr Rockets 26:188-195

9. Yurchenko D, Alevras P (2013) Dynamics of the N-pendulum and its application to a wave energy converter concept. Int J Dyn Control 1(4):290-299

10. Dai L, Wang X (2013) Accuracy and reliability of P-T method in studying the responses of nonlinear dynamic systems. ASME J Comput Nonlinear Dyn. doi:10.1115/1.4026895

11. Dai L (2008) Nonlinear dynamics of piecewise constant systems and implementation of piecewise constant arguments. World Scientific Publishing Co., New Jersey

12. Dai L, Singh MC (1997) Diagnosis of periodic and chaotic responses in vibratory systems. J Acoust Soc Am 102(6):33613371 